\title{
Photobiomodulation in Temporomandibular Dysfunction: a Systematic Review
}

\author{
A. M. Pimentel de França ${ }^{1}$, A. Gonçalves Borges ${ }^{1}$, R. Boff Daitx², R. M. Ferrer ${ }^{3}$, \\ M. Baptista Dohnert ${ }^{1}$, J. L. Q. Durigan ${ }^{4}$ \\ 1 Research, Teaching and Extension Laboratory in Orthopedic Trauma Physiotherapy (LAPEFITO), Gurupi \\ University (UnirG), Gurupi, Torres, Brazil \\ 2 Lutheran University of Brazil (Ulbra), Brazil \\ 3 Centro Universitário do Norte (Uninorte), Brazil \\ ${ }^{4}$ Postgraduate Program in Health Sciences (PPGCR), University of Brasília (UnB), Brazil
}

\section{CORRESPONDING AUTHOR:}

Marcelo Baptista Dohnert

Teaching and Extension Laboratory

in Orthopedic Trauma Physiotherapy

(LAPEFITO)

Gurupi University

Rua Joaquim Batista de Oliveira 355

Vila Alagoana

Fernando Osório av. 130 / 402 C

Pelotas, RS, Brazil

E-mail: mdohnert@unirg.edu.br

\section{DOI:}

10.32098/mltj.03.2021.11

LEVEL OF EVIDENCE: $1 \mathrm{~A}$

\begin{abstract}
SUMMARY
Background. Temporomandibular disorder (TMD) is defined as a set of painful or dysfunctional conditions involving masticatory muscles and the temporomandibular joint (TMJ). Photobiomodulation (PBM) is a non-invasive and non-pharmacological therapy method that can prove to be an alternative treatment and generate benefits for the treatment of TMD-related pain.

Methods. The authors conducted a systematic literature review to assess the effectiveness of photobiomodulation (PBM) in the treatment of TMD. Selection criteria included: 1) studies in human subjects, 2) articles without language restriction, and 3) placebo versus control randomized clinical trials. The review covered articles published over the past five years in the databases PubMed, Cochrane Library, Science Direct, and PEDro. Methodological quality assessment followed the PEDro criteria.

Results. A total of 12 articles were included in the review. In these studies, photobiomodulation reduced myogenic and arthrogenic pain. The predominant sites of application of PBM were either predetermined or painful areas of the masseter and temporal muscles and the TMJ. The application of infrared spectrum radiation (wavelength ranging from 790 to $905 \mathrm{~nm}$ ), with low doses of energy per application point ( 3 to $8 \mathrm{~J} /$ $\mathrm{cm}^{2}$ ), three times a week for 8 to 12 weeks, showed the best results.

Conclusions. Based on the results of this review, it is risky to draw definitive conclusions about the effectiveness of PBM for the treatment of TMD.
\end{abstract}

\section{KEY WORDS}

Temporomandibular Joint Disorders; laser therapy; low intensity light therapy; facial pain; lasers.

\section{BACKGROUND}

Temporomandibular disorder (TMD) is defined as a set of painful or dysfunctional conditions involving masticatory muscles and the temporomandibular joint (TMJ) (1-3). Muscle dysfunctions are the most prevalent conditions (4). The pain reported by patients with TMD is usually felt in the preauricular region, masticatory muscles, and the temporomandibular region (4). The signs and symptoms are diverse and may include difficulties in chewing, speech, and other orofacial functions (3). Notwithstanding, the most frequent symptoms include tenderness in the masticatory muscles, pain in one or both TMJs, and limited mandibular joint mobility (2). About 40 to $75 \%$ of the population shows at least one TMD sign throughout their lives (4). Although TMD occurs in both sexes, women are more susceptible to temporomandibular disorders, with proportions ranging from two to six women for each man (3). The most prevalent age range varies between 20 and 40 years (3). Poor posture, occlusal problems, harmful oral habits, and other clinical abnormalities may lead to TMD, which can also correlate with psychological factors (2). Among these, reports include anxiety, depression, and sleep bruxism $(5,6)$.

There are several conservative treatment options for TMD reported in the literature (7). Among these options we can mention myorelaxative splints in isolation or associated with 
physical therapies, acupuncture, cognitive behavioral therapies and hyaluronic acid injections (7). Photobiomodulation $(\mathrm{PBM})$ is a noninvasive and nonpharmacological therapy method that has shown great benefits for the treatment of TMD-related pain (8). This method uses light radiation within the infrared and visible red light spectrum of electromagnetic waves (8). Whether through low level laser or light emitting diode (LED), PBM is one of the most used noninvasive therapeutic methods by health professionals, such as physiotherapists and dentists, for the treatment of TMD (9). Phototherapy has been used more and more in the field of dentistry in the last thirty decades for its analgesic, anti-inflammatory, and biostimulant effect (10). However, it is not yet fully defined what these effects are and how they are best produced considering the different parameters of PBM used and addressed in the current literature.

Thus, we conducted a search in the scientific literature through a critical analysis of the effectiveness of photobiomodulation in temporomandibular disorder.

\section{MATERIALS AND METHODS}

This systematic review was conducted according to the PROSPERO protocol, registered under the number CRD 42020205039. This study meets the ethical standards of Muscles, Ligaments and Tendons Journal (11).

\section{Inclusion and exclusion criteria}

The review included randomized clinical trials (RCTs) from the last five years, which addressed the use of photobiomodulation with light emitting diode (LED) or low intensity laser for chronic myogenic and/or arthrogenic pain. The studies should provide a description of the type of LED or laser used, application time, and administration parameters. There was no language restriction. As a comparator, different types of laser, placebo laser, and no treatment (control) were accepted. The main outcome measures were chronic myogenic and/ or arthrogenic temporomandibular pain, mandibular range of motion in patients with TMD, and joint function.

\section{Search strategy}

Four electronic databases were searched (Cochrane Library, PubMed, PEDro, and Science Direct) from June to November 2020. The search strategies are described in table I. Thus, from the published findings, we gathered the most relevant data for the construction of this study (table I).

\section{Literature review and eligibility criteria}

To determine the eligibility of the identified articles, two of the five collaborators (AMPF, AGB, RBD, RF, MBD) inde- pendently selected titles, abstracts, and made the last analysis of the titles and contents. Any disagreement between authors on the study eligibility was resolved by discussion and adjudication by a third reviewer (MBD) as necessary.

\section{Risk of bias}

Risk of bias was assessed using the PEDro scale (12). Two authors independently assessed the risk of bias. Disagreements were resolved by discussion and adjudication by the lead author (MBD) as necessary. The results of the methodological quality of the RCTs included in the review are shown in table II.

\section{Study analysis}

Two of the following five collaborators (AMPF, AGB, RBD, $\mathrm{RF}, \mathrm{MBD}$ ) extracted data on sample characteristics, intervention design, and estimates of the effect of PBM in each study. The studies were described in terms of trial design (sample size, follow-up period, PEDro score), sample characteristics (population, allocation groups), and intervention components (PBM application site, application time, wavelength, treatment frequency, power of the equipment, dosimetry, outcome measures, and outcomes). Estimates of the effect of $\mathrm{PBM}$ on TMJ symptoms and function were extracted from each clinical trial.

\section{RESULTS}

The electronic search resulted in 120 articles (figure 1). After screening, 103 eligible RCTs were excluded for not meeting the eligibility criteria and five for duplication, resulting in 12 trials that provided data and were included in the review. The characteristics of these trials are shown in table III.

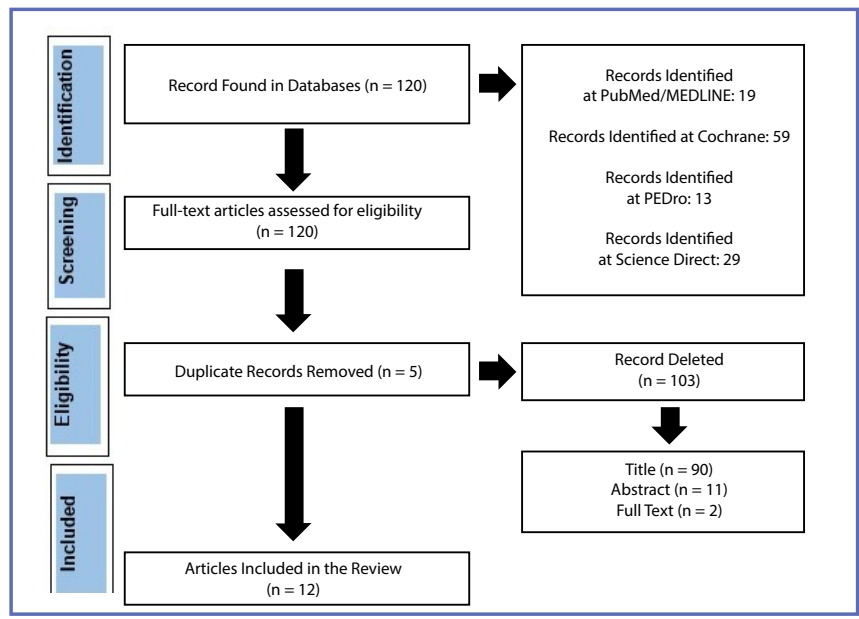

Figure 1. Flowchart with the search strategy used to search tor articles. 
Table I. Flowchart of the literature search strategy.

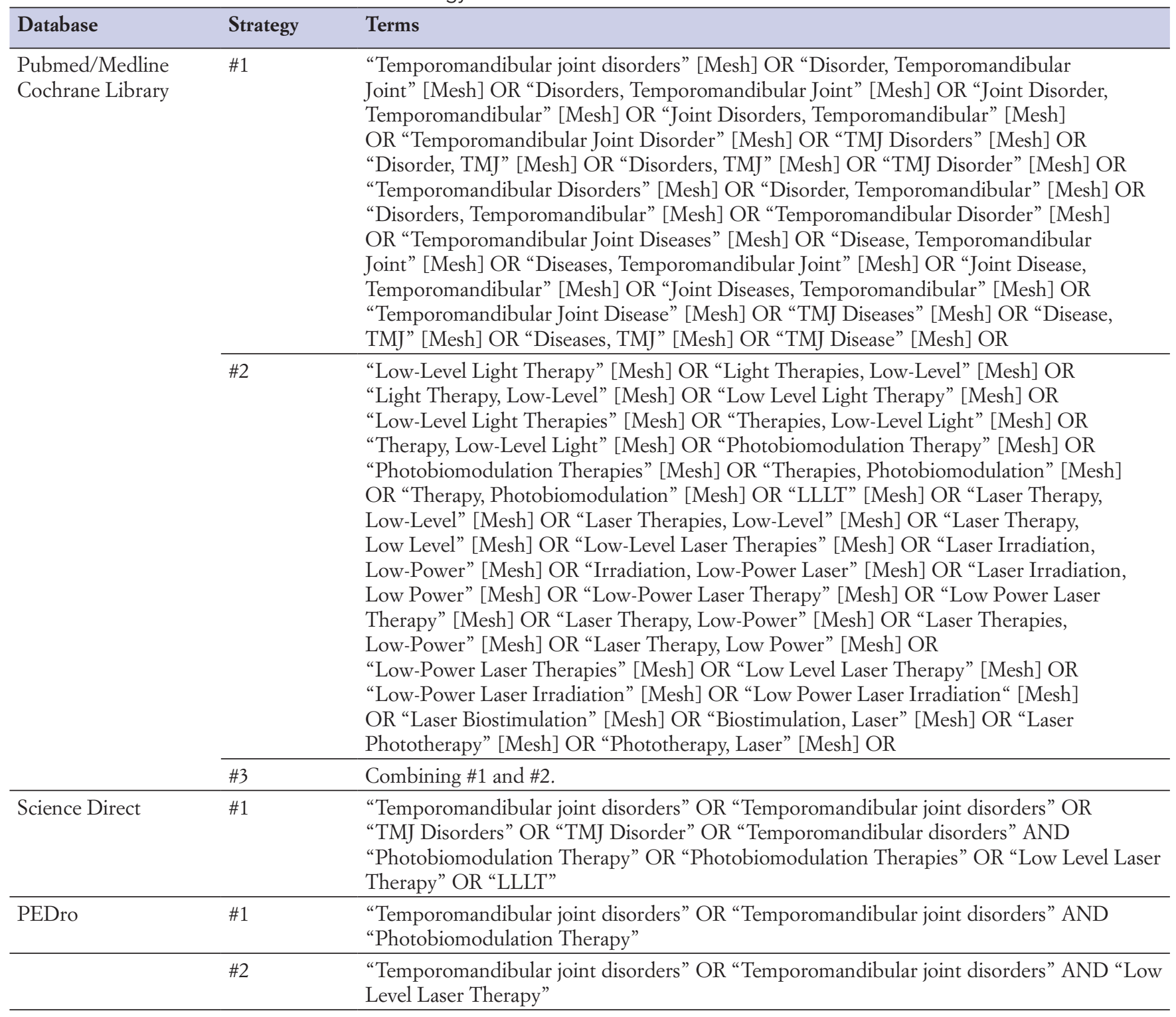




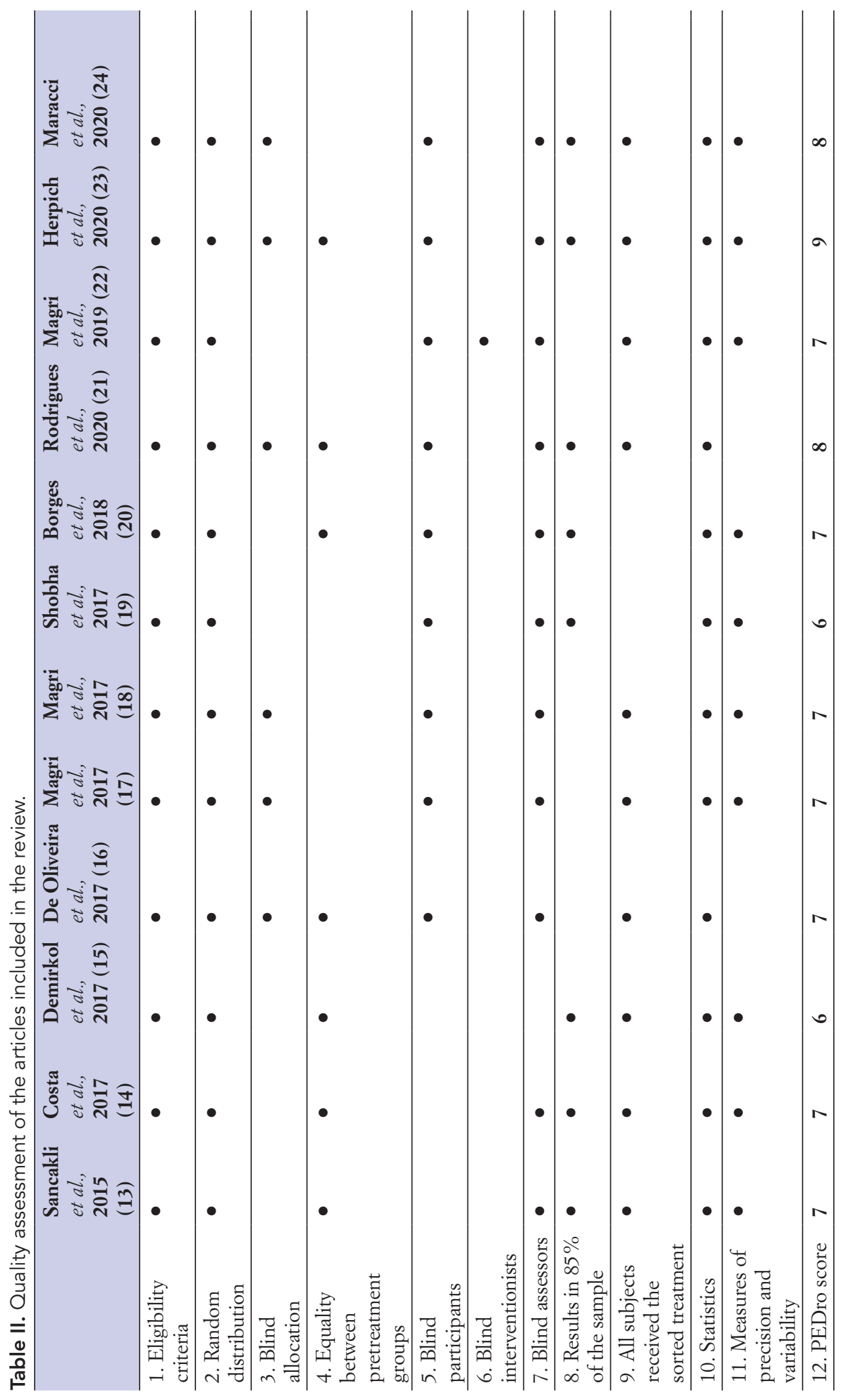




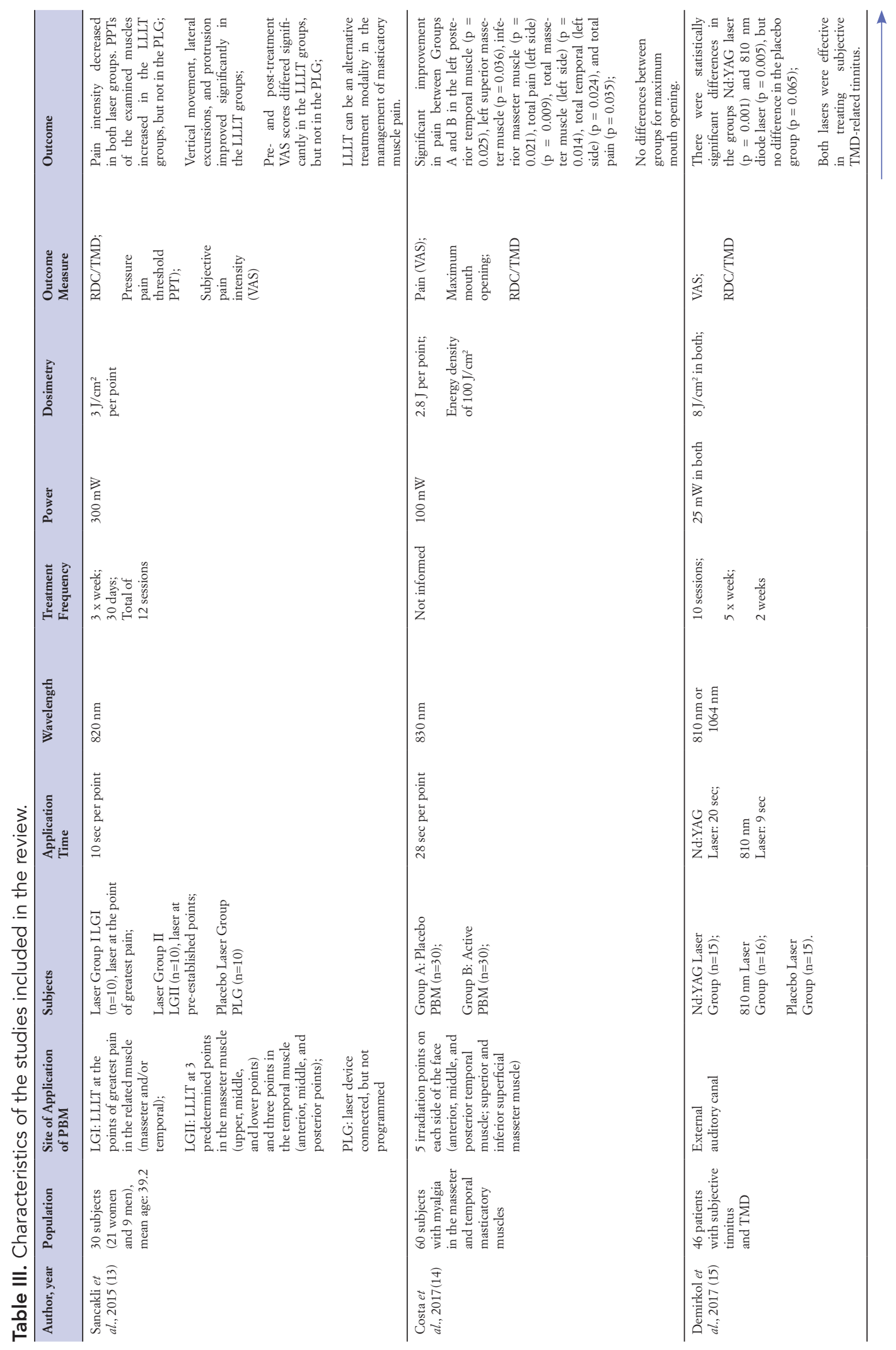

Muscles, Ligaments and Tendons Journal 2021;11 (3) 


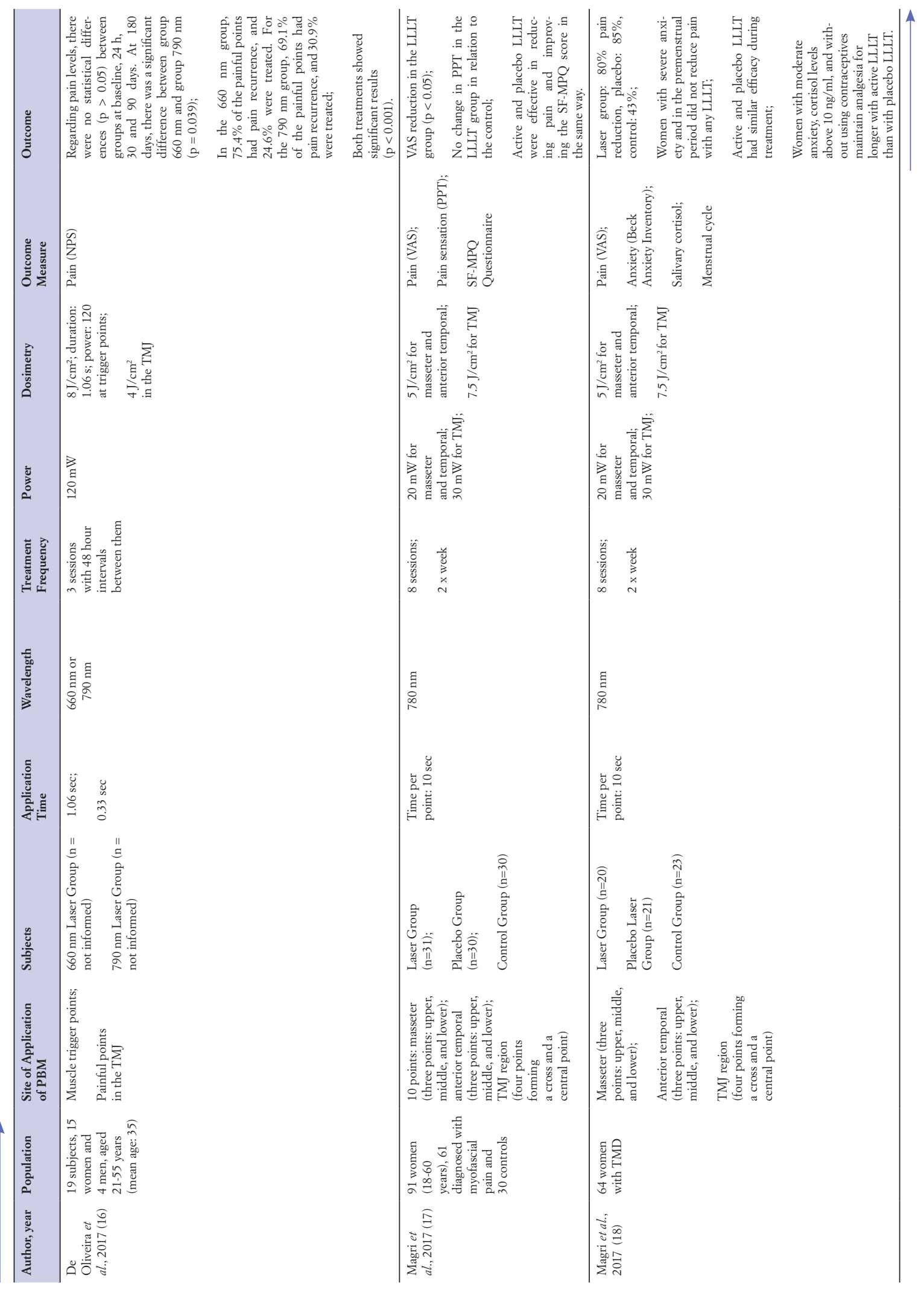




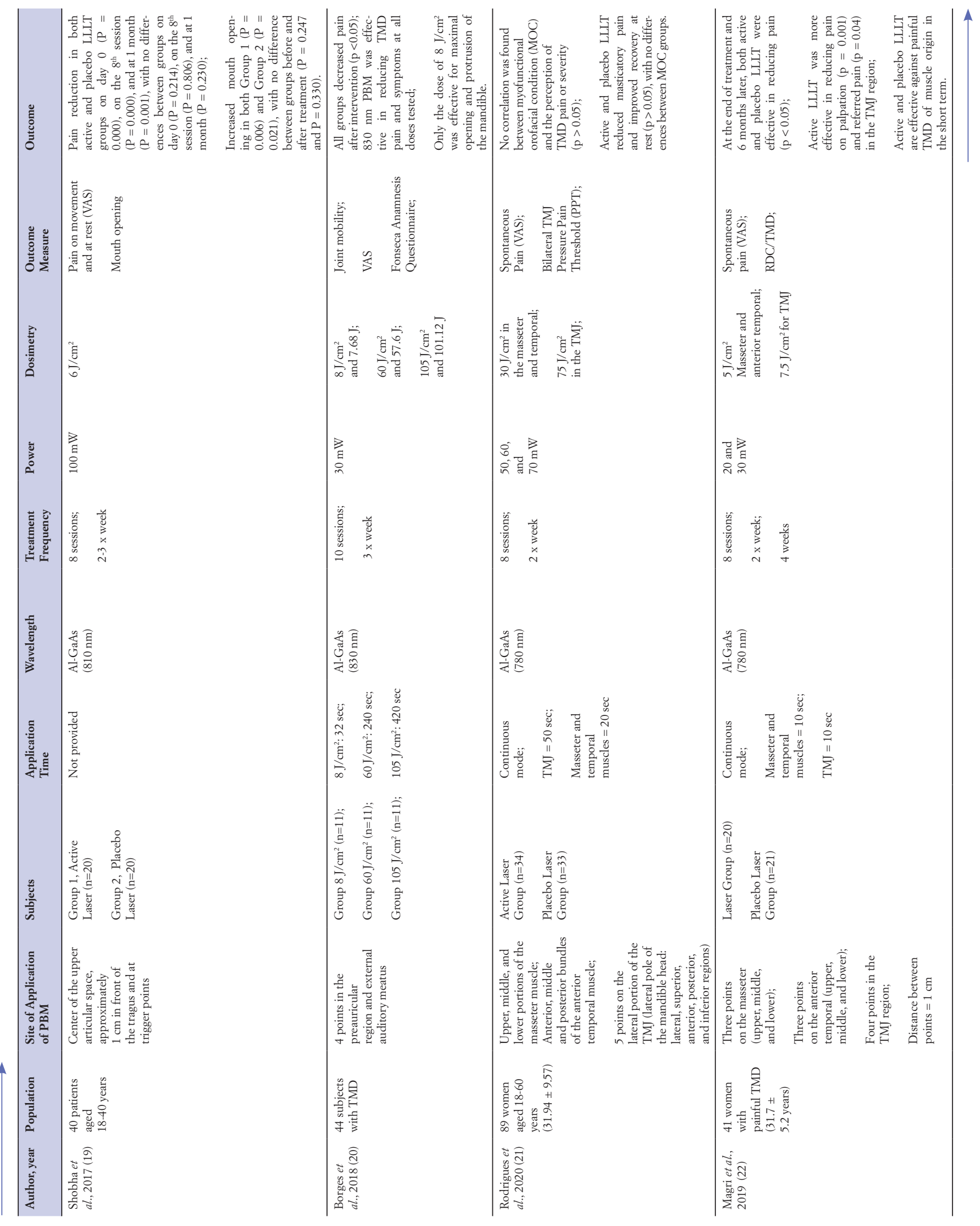




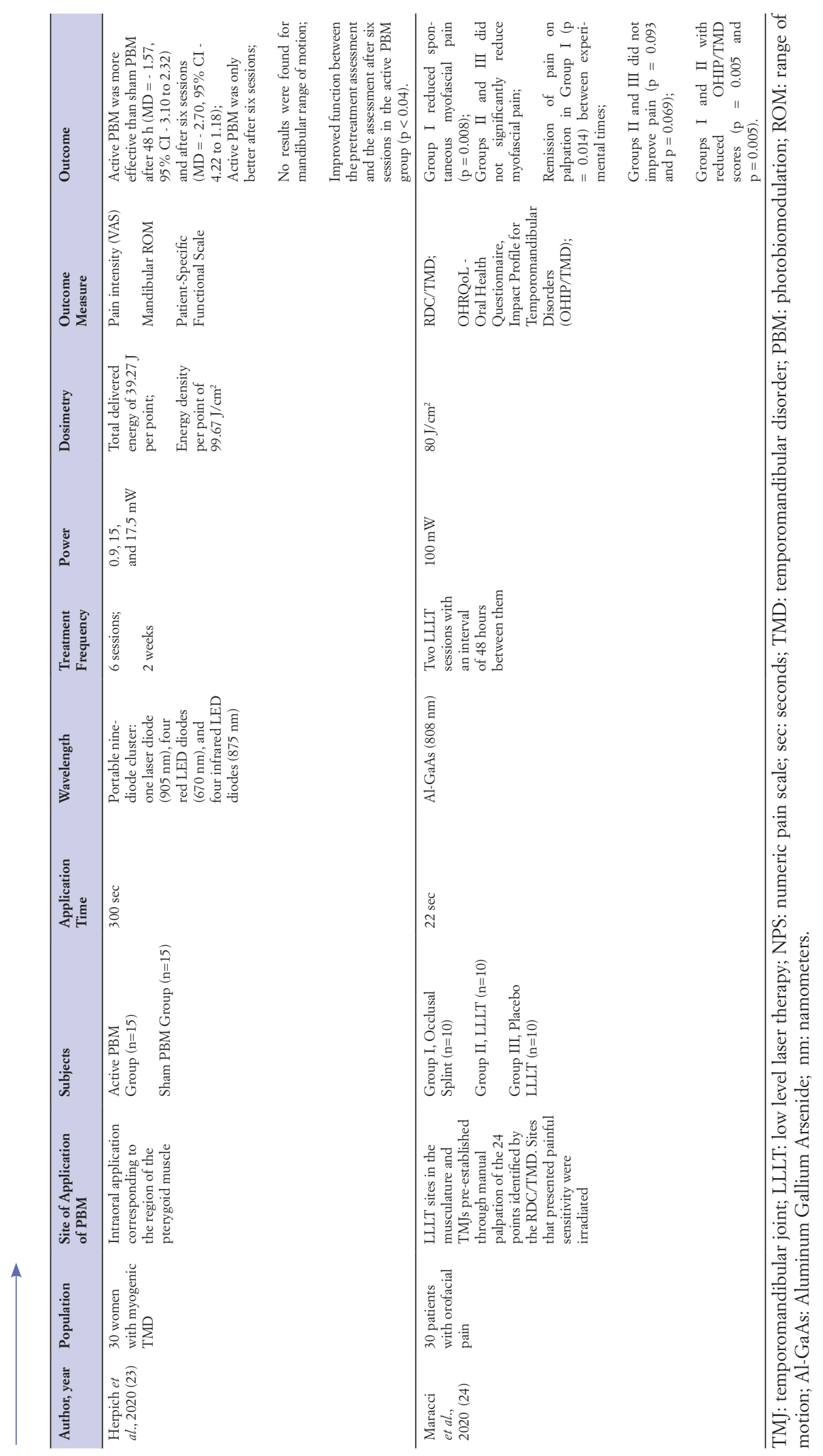


Table IV. Suggested protocol by the authors in TMD approach using FBM.

\begin{tabular}{ll}
\hline \multicolumn{1}{c}{ Parameter } & Suggestion \\
\hline Laser type & AsGaAl or its combination with visible red laser (LEDs). \\
Wavelength & 780 at $803 \mathrm{~nm}$ or this combined at $660 \mathrm{~nm}$ \\
Radiation power & 100 to $300 \mathrm{~mW}$ \\
Energy $(\mathrm{J})$ & 2 to $6 \mathrm{~J}$ per point \\
Energy density & 3 to $8 \mathrm{~J} / \mathrm{cm}^{2}$ per application point. \\
Application location & Pain points in the masseter and temporal muscles and in TMJ \\
Frequency & 8 to 12 sessions 3 times a week \\
\hline
\end{tabular}

TMD: temporomandibular disorder; AsGaAl: Aluminum Galium Arsenide; Nm: namometers; J: joule; TMJ temporomandibular joint.

\section{Site of application of photobiomodulation}

Among the twelve selected studies, seven applied PBM to the masseter muscle and temporal muscle in association with application to the TMJ $(16-19,21,22,24)$. The most used sites for irradiation were predefined points on the belly of the upper, middle and lower masseter muscle and on the anterior, middle and posterior temporal muscle $(17,18,21,22)$. Another three studies applied PBM to the trigger points of muscle pain (16, 19, 24). Only one study used PBM on the external auditory canal (15). Two studies used PBM only on the muscle belly of the masseter and temporal muscles $(13,14)$. One study used $\mathrm{PBM}$ at defined points in the TMJ region (preauricular region and external auditory meatus) (20). Finally, a study used PBM intraorally on the belly of the pterygoid muscle (22).

\section{Number and duration of photobiomodulation applications}

Most studies used PBM treatment twice a week for eight sessions, totaling four weeks of intervention $(17-19,21,22)$. One study did not report the time and duration of treatment (14). The other studies included different protocols such as three times a week in a total of twelve sessions (13), ten sessions twice a week (15), three sessions with an interval of 48 hours between them (16), three times a week for ten sessions (17), three times a week for six sessions (23), and two sessions with an interval of 48 hours between them (24).

\section{Photobiomodulation characteristics}

Five studies used laser in the $800-830 \mathrm{~nm}$ range $(13,14,19,20$, 24). In these studies, the power ranged from 30 to $300 \mathrm{~mW}$, and the energy density per point ranged from $2.8 \mathrm{~J} / \mathrm{cm}^{2}$ to 105 $\mathrm{J} / \mathrm{cm}^{2}$. Four studies used laser irradiation in the range of 780 $\mathrm{nm}(17,18,21,22)$. In these studies, the power ranged from 20 to $70 \mathrm{~mW}$, and the energy density per point was 5 to $75 \mathrm{~J} / \mathrm{cm}^{2}$. Two studies compared different irradiation wavelengths, one comparing the ranges $810 \mathrm{~nm}$ and $1064 \mathrm{~nm}$ (14) and the other comparing the ranges $660 \mathrm{~nm}$ and $790 \mathrm{~nm}$ (16). The power ranged from $25 \mathrm{~mW}(1064 \mathrm{~nm})$ to $120 \mathrm{~mW}(660$ and $790 \mathrm{~nm}$ ) (16), with dosimetry ranging from 3 to $8 \mathrm{~J} / \mathrm{cm}^{2}$. Finally, only one study used a laser cluster with irradiation spectra of 670,875 , and $905 \mathrm{~nm}$, with power ranging from 0.9 to $17.5 \mathrm{~mW}$ and energy from 39.27 to $99.67 \mathrm{~J}$ (24).

\section{Outcome measures}

The main outcome measure used in the studies was pain. Ten studies used the visual analog scale (VAS) in their outcome measures $(13-15,17-23)$. Four studies used the pressure pain threshold (PPT) $(13,17,18,21)$. Only one study used the Numeric Pain Scale (NPS) (16).

Four studies evaluated mouth opening mobility $(14,19,20$, 23). To assess function, five studies used the RDC/TMD questionnaire $(13-15,22,24)$ and one used the OHIP/TMD (24) questionnaire. In addition to these main measures, a study assessed quality of life using the SF-MPQ questionnaire (17) and a study assessed the level of anxiety, salivary cortisol, and menstrual cycle (18).

\section{Outcomes}

All ten studies that assessed pain showed its significant improvement after PBM intervention (13-20, 22, 23). Two out of the four studies that assessed TMJ range of motion (ROM) found an improvement in this outcome $(13,20)$; in the study by Borges et al. (20), only the dosimetry of $8 \mathrm{~J} / \mathrm{cm}^{2}$ improved the variable. Finally, function improved only in the study by Demirkol et al. (15).

\section{Risk of bias}

The mean PEDro score obtained in the studies was 7.2. Of the included studies, all met the random allocation criteria $(100 \%)$. Six did not provide information on blind allocation criteria $(50 \%)$. Eleven studies met the criteria for blind 
assessment $(91.7 \%)$, nine for blind participants (75\%), and one for blind interventionist $(8.3 \%)$. Eight studies met the criterion for the descriptions of losses and exclusions, $(66.7 \%)$. The criteria for the analysis by treatment intention were met in ten studies $(83.3 \%)$. The results of the risk of bias assessment are shown in table II.

\section{DISCUSSION}

Temporomandibular disorder (TMD) is defined as a set of disorders originating in the temporomandibular joint region, masticatory muscles, and related structures $(9,13$, $14,16,17,18,22)$. These disorders correlate with changes in emotional state, behavioral changes, and social interactions $(9,13,14,16,17,20,22)$. The etiology is multifactorial and parafunctional (15). This condition is usually triggered by occlusal conditions, emotional stress, traumas, parafunctional habits (jaw tightness and bruxism), instability in maxillary and mandibular relations, joint laxity, comorbidity of rheumatic or musculoskeletal diseases (15). In this systematic review, we conducted a search in the scientific literature through a critical analysis of the effectiveness of PBM on TMD symptoms, aiming to verify what are the best parameters to achieve this effectiveness.

Photobiomodulation (PBM) is a noninvasive treatment technique used in the clinical practice of both physiotherapy and dentistry for pain relief and tissue regeneration. This technique has shown beneficial results in the TMD approach (16). Many clinical studies have investigated the effects of PBM on TMD, whether of myogenic or arthrogenic origin, through objective and subjective measures to assess pain in these regions $(13,24)$. Pain is a complex and multidimensional phenomenon based on the individual report of the lived experience. It is susceptible to a variety of factors that can interfere with its perception (21). However, other factors may be more relevant to the perception of pain intensity during mandibular function, such as joint diagnosis, pain catastrophizing, anxiety, previous painful experiences, among others (21).

Photobiomodulation is effective in the treatment of temporomandibular disorder, especially on pain, given its analgesic and anti-inflammatory effects. In this review, ten studies directly assessed the level of joint and muscle pain. All of them demonstrated positive effects of PBM. Pain reduction can be justified by the reduction in the expression of inflammatory cytokines, as well as by the stimulation of local microcirculation in the irradiated area $(16,23)$. Animal model studies have shown that PBM can reduce proinflammatory substances in TMJ (22). Notwithstanding, the analgesic effect may stem from different endogenous mechanisms such as the release of endogenous opioids, increased production of ATP, reduced production of prostaglandins (especially COX-2), reduced lymphatic metabolism, and secretion of histamine, kinins, and cytokines (16, 19, 22). In association, an increase in the release of endorphins and encephalins can lead both to the modulation of nociceptors and to changes in the transmission of painful nerve impulses $(14,16,22)$. The main mechanism of PBM, however, consists of metabolic activation through the stimulation of the mitochondrial respiratory chain $(14-16,19,22)$. This phenomenon increases vascularization and improves oxygen supply in hypoxic cells $(15,16,22)$. At the cellular level, PBM releases protons into the cytoplasm. This reduces the permeability of the channels to $\mathrm{Na}^{+}$and $\mathrm{K}^{+}$ions, decreasing the frequency of the action potential (15). It also accelerates the functional and morphological recovery of severely injured nerve tissues, including damaged cells in the auditory system, and provides therapeutic effects in patients with inner ear disorders $(14,15)$.

The effects of PBM on the control of moderate pain in patients with painful TMD are quite significant for the improvement of functional patterns of mandibular mobility. Eight of the included studies assessed TMJ function. However, only three of these showed improvement in these variables. The RDC/TMD criteria for Axes I and II have been widely used in clinical studies to characterize physical, psychological, and psychosocial factors associated with TMD. Likewise, the relationship between these factors and RDC/TMD has been suggested as a model for diagnosis and assessment of all clinical conditions of chronic pain (19). Failure to improve function in some studies can be attributed to kinesiophobia associated with the presence of pain rather than to the actual functional limitation of these patients with myofascial pain (18). Herpich et al. (23) state that the number of treatment sessions can influence functional improvement, and suggest a greater number of treatment sessions.

Photobiomodulation with infrared light was used in isolation in ten studies included in the review. Two studies used a combination of visible red and infrared laser. The effectiveness of PBM seems to be more pronounced when using infrared laser associated with application protocols involving higher levels of irradiation (energy density and/or power density), greater number of sessions, and higher frequency of application $(18,19)$.

It is noteworthy that, regarding the treatment of TMD, there is no consensus in the scientific literature on PBM dosimetry, application area, duration, and protocols. This hinders research standardization and comparison of results. In some studies, the technique used consists of applying the laser on 
the points of greatest pain. Others use laser at predetermined points. Most studies used a protocol with eight to twelve sessions, two to three times a week.

Maracci et al. (24) stated that infrared laser applications with an interval of 48 hours between sessions was not enough to remit painful symptoms. In comparison to the placebo treatment, the effects of PBM on the symptomatology of TMD patients can be mitigated after eight sessions (24).

Another important factor is that PBM is not a lasting and permanent treatment for TMD, as the results showed a high rate of pain recurrence. Identifying the etiology of TMJ disorders is extremely important to treat and prevent TMD symptoms. Using PBM in association with other treatment modalities (invasive or not), considering the clinical condition of each patient, seems to be the best alternative (16).

A placebo effect was found in four studies included in the review. This is defined as beneficial health outcomes not directly related to the biological effects of an intervention, but rather to an inert agent (18). Magri et al. (17) believe that patients with TMD are susceptible to placebo effects due to the psychological component involved. Moreover, the desire to feel better seems to influence physiological processes, thus leading to a favorable result (20). These effects correlate with beneficial cognitive effects such as stress reduction and modulatory mechanisms of mesolimbic and mesocortical areas related to the expected cure/ improvement generated by the proposed intervention (18). The brain mechanisms activated by the placebo effect are similar to those activated by drugs (18).

\section{REFERENCES}

1. Bitencourt GB, Gonçalves MLL, Kobayashi FY, et al. Administration of low-level laser on muscles of mastication following the induction of initial fatigue: protocol for a randomized, controlled clinical trial. Medicine 2018;97(26):e11340.

2. GogoY CHL, Motta LJ, Fernandes KPS, Mesquita-Ferrari RA, Deana AM, Bussadori SK. Effect of Low-Level Laser Therapy on Adolescents with Temporomandibular Disorder: A Blind Randomized Controlled Pilot Study. J Oral Maxillofac Surg 2015;73(4):622-9.

3. Ferreira CLP, Silva MAMRD, Felicio CMD. Signs and symptoms of temporomandibular disorders in women and men. CoDAS 2016;28(1):17-21.

4. Zotelli VL, Grillo CM, Gil ML, Wada RS, Sato JE, Sousa MDLRD. Acupuncture Effect on Pain, Mouth Opening Limitation and on the Energy Meridians in Patients with Temporomandibular Dysfunction: A Randomized Controlled Trial. JAMS 2017;10 (5):351-9.

5. Zavala CEM, Rosas ASC, Ancona PEL, Mendiburu JC. Disfunción temporomandibular y depresión en adolescentes
The psychosomatic context of the patient during any therapy can change the biochemical and neural pathway, generating satisfactory clinical results very similar to those of the proposed effective intervention, although without any intervention (18). The response to placebo differs substantially depending on the characteristics of the pain, with chronic pain being much more susceptible to positive responses than acute pain (17).

\section{CONCLUSIONS}

Based on the results of this review, it is risky to draw definitive conclusions about the effectiveness of PBM for the treatment of TMD. The studies have shown many methodological differences, mainly in relation to the number, location, and duration of laser applications, as well as in relation to the characteristics of the laser beam (wavelength, frequency, output, dosimetry). It is therefore not possible to standardize guidelines for effective treatment with PBM. However, it seems clear to us that the use of PBM in the infrared spectrum or its association with red laser, with low energy $(\mathrm{J})$ and energy density $\left(\mathrm{J} / \mathrm{cm}^{2}\right)$ applications, in the range of 3 to $8 \mathrm{~J} / \mathrm{cm}^{2}$ per point of application, with a total of eight to twelve sessions three days a week, produced more significant effects on pain relief. The effects of PBM on TMJ mobility and function are not yet clear.

\section{CONFLICT OF INTERESTS}

The authors declare that they have no conflict of interests.

de ascendencia maya. / Temporomandibular dysfunction and depression in adolescents of Mayan zancestry. J Appl Oral Sci 2020;77(3):127-34.

6. Leite CMA, Barbosa SA JS, Conti PCR. How psychosocial and economic impacts of COVID-19 pandemic can interfere on bruxism and temporomandibular disorders. JAOS 2020;28:e20200263.

7. Feitoza CC, de Lemos Menezes P. Prognosis of conservative treatment in individuals with temporomandibular disorders and tinnitus: a systematic review. Muscles Ligaments Tendons J 2018;8(1):123-4.

8. Sobral APT, Godoy CLH, Fernandes KPS, et al. Photomodulation in the treatment of chronic pain in patients with temporomandibular disorder: protocol for cost-effectiveness analysis. BMJ 2018;8(5):e018326.

9. Herpich CM, Junior ECPL, Amaral AP, et al. Effects of phototherapy on muscle activity and pain in individuals with temporomandibular disorder: a study protocol for a randomized controlled trial. Trials 2014;15:491. 
10. Franco WF, Galdino MVB, Capeletti LR, et al. Photobiomodulation and Mandibular Advancement Modulates Cartilage Thickness and Matrix Deposition in the Mandibular Condyle. Photobiomodul Photomed Laser Surg 2020;38 (1):3-10.

11. Padulo J, Oliva F, Frizziero A, Maffuli N, Muscles, Ligaments and Tendons Journal - Basic principles and recommendations in clinical and field Science Research: 2018 update. Muscles Ligaments Tendons J 2018;8(3):305-7.

12. Maher CG, Sherrington C, Herbert RD, et al. Reliability of the PEDro scale for rating quality of randomized controlled trials. Phys Ther 2003;83:713-21.

13. Sancakli E, Röhlig BG, Balik A, Öngul D, Kipirdi S, Keskin H. Early results of low-level application for masticatory muscle pain: a double-blind randomized clinical study. BMC Oral Health 2015;23;15(1):131.

14. Costa SAP, Florezi GP, Artes GE, Gallo RT, Costa JR, Freitas PM, et al. The analgesic effect of photobiomodulation therapy $(830 \mathrm{~nm})$ on the masticatory muscles: a randomized, doubleblind study. Braz Oral Res 2017;31:e107.

15. Demirkol N, Usumez A, Demirkol M, Sari F, Alkaboy C. Efficacy of Low-Level Laser Therapy in Subjective Tinnitus Patients with Temporomandibular Disorders. Photomed Laser Surg 2017;35(8):427-31.

16. De Oliveira DWD, Lages FS, Guimarães RC, et al. Do TMJ symptoms improve and last across time after treatment with red $(660 \mathrm{~nm})$ and infrared $(790 \mathrm{~nm})$ low level laser treatment (LLLT) ? A survival analysis. Lasers Med Sci 2017;32(2):419-28.

17. Magri LS, Carvalho VA, Rodrigues FCC, Bataglion C, Panissi CRAL. Non-specific effects and clusters of women with painful
TMD responders and non-responders to LLLT: double-blind randomized clinical trial. Lasers Med Sci 2018;33(2):385-92.

18. Magri LV, Carvalho VM, Rodrigues FCC, Bataglion C, Panissi CRAL. Effectiveness of low-level laser therapy on pain intensity, pressure pain threshold, and SF-MPQ indexes of women with myofascial pain. Lasers Med Sci 2017;32(2):419-28

19. Shoba R, Narayanan VS, Pai J. Low-level Laser Therapy: A Novel Therapeutic Approach to Temporomandibular Disorder - A Randomized, Double-blinded, Placebo-controlled Trial. Indian J Dent Res 2017;28(4):380-7.

20. Borges RMM, Cardoso DS, Flores BC, Luz RD, Machado CR, Cerveira GP, et al. Effects of different photobiomodulation dosimetries on temporomandibular dysfunction: a randomized, double-blind, placebo-controlled clinical trial. Lasers Med Sci 2018;33(9):1859-66.

21. Rodrigues CA, Melchior MO, Valencise Magri L, Mazzetto MO. Can the severity of orofacial myofunctional conditions interfere with the response of analgesia promoted by active or placebo low-level laser therapy? Cranio 2020;38(4):240-7.

22. Magri LS, Bataglion C, Panissi CRAL. Follow-up results of a randomized clinical trial for low-level laser therapy in painful TMD of muscular origins. Cranio 2019;4:1-8.

23. Herpich CM, Junior ECPL, Politti F, et al. Intraoral photobiomodulation diminishes pain and improves functioning in women with temporomandibular disorder: a randomized, sham-controlled, double-blind clinical trial. Lasers Med Sci 2020;35(2):439-45.

24. Maracci LM, Stasiak G, Chami VO, et al. Treatment of myofascial pain with a rapid laser therapy protocol compared to occlusal splint: A double-blind, randomized clinical trial. Cranio 2020;3:1-7. 